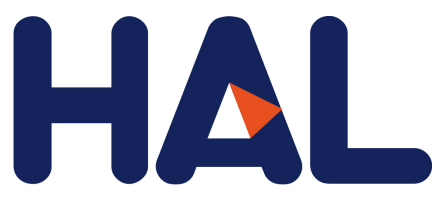

archives-ouvertes

\title{
THE GROWTH OF THE Pt/Si (111) $7 \times 7$ INTERFACES AS PROBED BY Pt L3 SEXAFS
}

\author{
G. Rossi, D. Chandesris, P. Roubin, J. Lecante
}

\section{To cite this version:}

G. Rossi, D. Chandesris, P. Roubin, J. Lecante. THE GROWTH OF THE Pt/Si (111) 7 x 7 INTERFACES AS PROBED BY Pt L3 SEXAFS. Journal de Physique Colloques, 1986, 47 (C8), pp.C8-521C8-524. 10.1051/jphyscol:1986897 . jpa-00226228

\section{HAL Id: jpa-00226228 \\ https://hal.archives-ouvertes.fr/jpa-00226228}

Submitted on 1 Jan 1986

HAL is a multi-disciplinary open access archive for the deposit and dissemination of scientific research documents, whether they are published or not. The documents may come from teaching and research institutions in France or abroad, or from public or private research centers.
L'archive ouverte pluridisciplinaire HAL, est destinée au dépôt et à la diffusion de documents scientifiques de niveau recherche, publiés ou non, émanant des établissements d'enseignement et de recherche français ou étrangers, des laboratoires publics ou privés. 


\title{
THE GROWTH OF THE Pt/Si (111) $7 \times 7$ INTERFACES AS PROBED BY Pt $\mathrm{L}_{3}$ SEXAFS
}

\author{
G. ROSSI, D. CHANDESRIS, P. ROUBIN and J. LECANTE \\ LURE, (Lab. CNRS, CEA, MEN), Université Paris-Sud, \\ F-91405 Orsay Cedex, France
}

\section{Abstract}

SEXAFS and XARS on the Pt, L2, 3 edges for submonolayer and monolayer coverages on Si (111) $7 \times 7$ allow to recognize the growth mode of the $\mathrm{Pt} / \mathrm{Si}$ silicide like interface, at room temperature. Chemisorption into sixfold interstitial sites in the top $\mathrm{Si}$ (111) double layer is followed by intermixing, and likely nucleation of Pt2Si coordinated clusters.

Direct information on the local environment of Pt atoms deposited onto Si (111) $7 \times 7$ surfaces is obtained from SEXAFS, allowing the discussion of the growth phenomenology of the Pt/Si. (111) interfaces formation process, in combination with new XARS, and published photoemission and Auger results $(1,2)$.

The chemisorption site derived from the submonolayer interface (0.8 ML Pt onto $\mathrm{Si}(111) 7 \times 7$ ) is the six fold interstitial lying midway between the top and the second Si layer, with a PtSi first nearest neighbour distance of $2.48+0.03$, and a consequent deformation of the $S i$ top doublelayer. The identification of the six fold interstitial site for chemisorbed Pt, unambiguously derived from the first distance and coordination number appearing in the SEXAFS, confirms that this site is a priviledged high coordination near surface site, since Ni (3), Co (4) and Ag (5) are also found to chemisorb in such site when deposited and/or annealed on Si (111) substrates. Pt chemisorption into interstices induces a strain of the Si host lattice to allow for the measured 2.48 \& bonding distance. The deformation of the Si sixfold cage can be observed from the SEXAFS data by considering the peaks in the Fourier transform beyond the first nearest neighbour peak. An EXAFS simulation for the sixfold interstitial position of $P t$ in the top $S i$ double layer, with the experimental phase shift (from $P t S i O)$ and backscattering amplitudes (1), reproduces the peaks beyond the first one (set by the experimental value) up to the fifth Pt-Si 
distance. The agreement of the simulation with the submonolayer data, beyond confirming the site assignement (simulations for all other possible surface and interstitial sites did not compare well both with the first and the higher FT peaks), indicates that the six Si first neighbours are displaced both vertically and outward with respect to their unperturbed positions. The top Si layer appears to be locally lifted by $0.58 \mathrm{~A}$, and all six Si atoms move outward by 0.15 A. The distance between the second and third Si layers remains unperturbed (2.35 \&) while parallel to the surface the Si-Si distances are little compressed. The perturbation of the Si lattice parallel to the surface is therefore of medium range, which explains the changes in the $S i$ valence band density of states observed with surface sensitive photoemission for submonolayer Pt chemisorption (2). The error bars on the Si-Si displacements deduced from the high distance SEXAFS information are relatively large, and some of the details may seem coincidental, nevertheless the simulation of Fig. 1 does not contain arbitrary parameters other than the geometry of the site (independently ascertained) and the experimental phase and amplitudes (assumed to be valid also for higher distances than the first), and it is the best approximation to the data. Not allowing for the lateral expansion would impose a vertical displacement of the top three atoms by $1.44 \mathrm{~A}$, and a second Pt Si distance $0.31 \mathrm{~A}$ longer than observed.

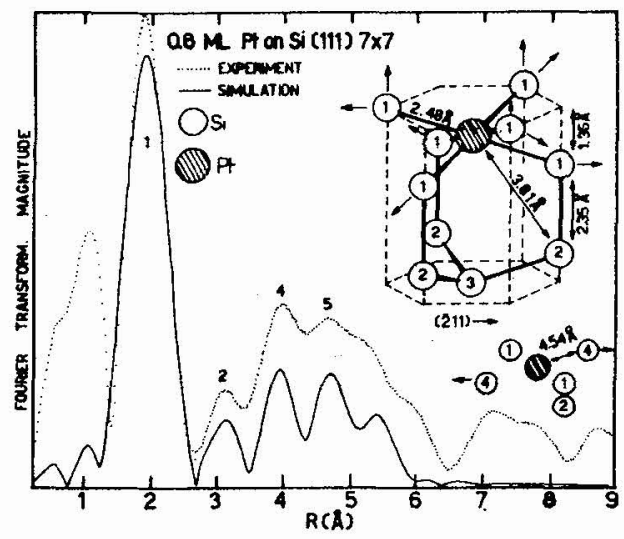

Figure 1 : Fourier transformation (Ft) of Pt L3 SEXAFS data for $0.8 \mathrm{ML}$ Pt onto $s i(111) 7 \times 7$ (dots) and simulation of the sixfold interstitial site of the top Si(111) double-layer. Peaks 2,4,5 correspond to simulated $\mathrm{Pt}-\mathrm{Si}$ distances as detailed in the schematic structural model for chemisorbed Pt.

The SEXAFS results for $2.5 \mathrm{ML}$ Pt/Si (111) show a prominent first distance frequency, corresponding to $2.49+0.03 \mathrm{~A} \mathrm{PtSi}$ distance, with a reduced coordination number. No higher distance contributions are observed out of the noise. This stage with reduced order and coordination describes the intermixing stage of the interface, with Pt occupying probably inequivalent sites in the Si distorted matrix. The bonding configuration, as monitored by XARS (1) and photoemission (2), is already described by the molecular orbital bonding-antibonding Pt $5 \mathrm{~d}-\mathrm{Si} 3 \mathrm{p}$ hybrid states.

Fourier transformed SEXAFS data for $5 \mathrm{ML}$ Pt/Si are presented in Fig. 2. The first distance peak (A) indicates an almost unvaried PtSi first distance, but a 
sharp second peak (B) appears out of the noise. This peak is attributed to Pt-Pt backscattering, but, due to the almost perfect phase opposition of the Pt-Si and Pt-Pt EXAFS oscillations, cancellation of EXAFS amplitude occurs in the region of maximum overlap of the Pt-Si and Pt-Pt contributions, and the peak $B$ appears therefore unphysically narrow. The two peaks must be analyzed together as a two shell problem. The solid line is the best simulation of the data, reproducing the peaks position and widths, although not the relative amplitudes, and corresponds to a "local" $\mathrm{Pt} 2 \mathrm{Si}$ coordination : $4 \mathrm{Si}$ neighbours at $2.46 \mathrm{~A}$ and $4 \mathrm{Pt}$ neighbours at 2.78 凡. This would correspond to nucleation of silicide-like clusters within the PtSi solid solution intermixed interface region. At higher coverages the SEXAFS data contain unresolved Pt-Pt signal coming probably both from the silicide-like nuclei and from unreacted Pt clusters. The end product, after annealing at $600^{\circ} \mathrm{C}$ for several hours of the Pt-Si reaction is PtSi an othorombic compound whose EXAFS is reproduced in Fig. 3 along with a schematics of the projection along the $c$ axis of the structure (6). The interface formation mechanism found for $\mathrm{Pt} / \mathrm{Si}$ (111) differs from Ni/Si (111) which showed the direct epitaxial growth of $\mathrm{NiSI}_{2}$ by way of substitution of $\mathrm{Ni}$ in $\mathrm{Si}$ lattice positions, and rotation of the surface lattice. In the case of Pt silicides no cubic structures exist, $\mathrm{Pt}_{2} \mathrm{Si}$ is tetragonal, PtSi orthorombic, and the metastable $\mathrm{Pt}_{3} \mathrm{Si}$ is monoclinic. Therefore no direct epitaxy of
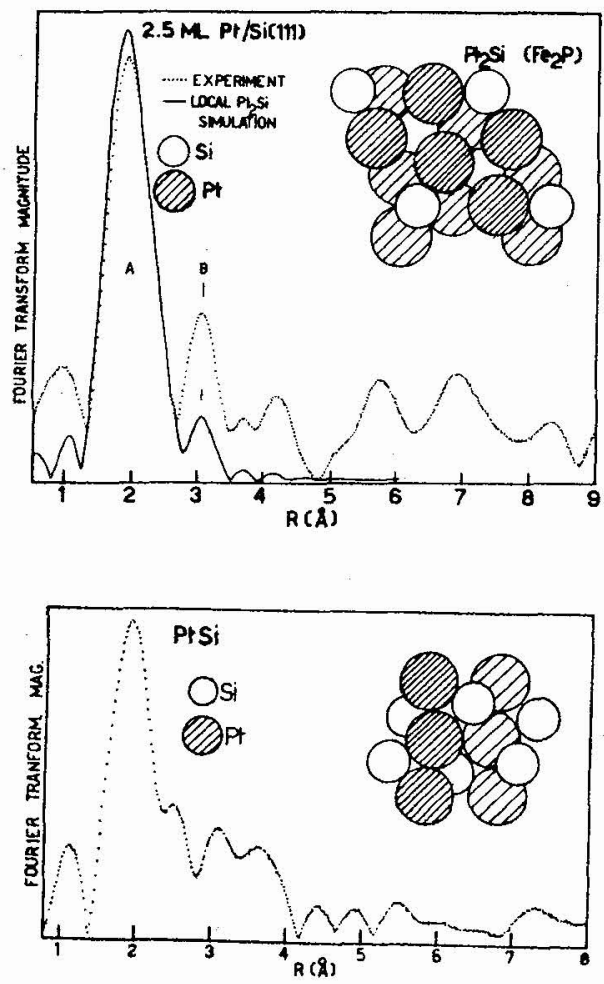

Figure 2 : Ft of the 5 ML Pt/Si(111) Pt L3 SEXAFS (dots) and simulation of a. local Pt2Si coordination, i.e. the first (Pt-Si) and second (Pt-Pt) shell of the structure shown in the top-right as seen along the e-axis.

Figure 3 : Ft of PtSi (epitaxially grown at $600^{\circ} \mathrm{C}$ ) and scheme of the PtSi structure as seen along the c-axis. 
a silicide on top of $S i(111)$ can be obtained, without major displacements of a very large number of atoms. In fact the nucleation of silicide-like clusters within an intermixed region seems to follow rather closely a bulk reaction phenomenology, after an early chemisorption stage dominated by the surface and near surface specific energetics. The $\mathrm{Pt} / \mathrm{Si}$ system has been considered for many reasons a prototype system of a reactive silicide like interfaces; the SEXAFS derived phenomenology seems to further define a silicide formation process which might be of wide generality.

\section{REFERENCES}

(1) G. ROSSI, D. CHANDESRIS, P. ROUBIN and J. LECANTE Phys. Rev. B unpublished; Surf. Sci. 168, 787 (1986)

(2) G. ROSSI, I. ABBATI, L. BRAICOVICH, I. LINDAU, W.E. SPICER Phys. Rev. B 25, 3627 (1982) and references therein

(3) F. COMIN, J. ROWE and P. CITRIN Phys. Rev. Lett. 51 2402 (1983)

(4) F. COMIN and P. CITRIN unpublished, private communication

(5) J. STOHR, R. JAEGER, G. ROSSI, T. KENDELEWICZ and I. LINDAU Surf. Sci. 134, 813 (1983)

(6) R.W.G. WYCKOFF Crystal structures, Krieger (1981) 